\title{
FORUM
}

\section{Graphical Methods of Finding the Sun's Azimuth and Elevation}

\author{
from D. H. Shinn \\ (Marconi's W.T. Company)
}

LikE Mr. R. S. Blicq (Journal I 5, 456) we have also been concerned in supplying details of the Sun's position to engineers on radar sites who use radiation from the Sun for, at present, two purposes:

(a) Measuring or checking the radiation pattern of the aerial in the vertica! plane.

(b) Lining up the aerial in azimuth or elevation.

For these purposes the required accuracy of computation is usually about \pm 0.1 in azimuth and elevation. Greater accuracy is never required, but less accuracy is sometimes adequate, up to $\pm \mathrm{I}^{\circ}$.

We have found that graphical methods appeal most to engineers, and that even when a full table of values is supplied to them they prefer to display this information in graphical form. We have accordingly evolved a simple method of constructing graphs of elevation against time, and azimuth against time.

In order to achieve the required accuracy of $\pm 00_{1}$ in elevation we have constructed a graticule of size $2 \mathrm{I}$ in. $\times 40$ in. A skeleton version of this is shown in Fig. I. The engineer has to know his latitude, and to find, from the Nautical Almanac or other source, the time of local noon (i.e. when the Sun is due south) and the mean declination, $\delta$, of the Sun during the period of observation, which usually lasts up to three hours. In order to be able to draw a graph of elevation against time, he has to compute some or all of the following quantities:

$$
\begin{aligned}
\mathrm{E}_{1} & =90^{\circ}-\lambda-\delta \\
\mathrm{E}_{5} & =90^{\circ}-\lambda+\delta \\
P & =\sin \mathrm{E}_{1} \\
Q & =\sin \mathrm{E}_{5} \\
M & =P+Q \\
\mathrm{E}_{2} & =-4 P+M \\
\mathrm{E}_{3} & =-4 P+2 M \\
\mathrm{E}_{4} & =-4 P+3 M
\end{aligned}
$$

He then draws a straight line on the graph through $E_{1}, E_{2}, E_{3}, E_{4}$ and $E_{5}$. This is a graph of $\theta_{t}$, the true elevation of the Sun, against apparent solar time (i.e. time adjusted so that the Sun is due south at $\mathrm{I} 2 \mathrm{~h}$. oom.) The abscissa has been marked in Fig. I with time during the morning; for afternoon observations the diagram must be turned upside down.

If the Nautical Almanac (or similar publication) is not available to the engineer at the radar station, it will be necessary to tell him the declination of the Sun and the time of local noon for the period of observation. He can then, however, 


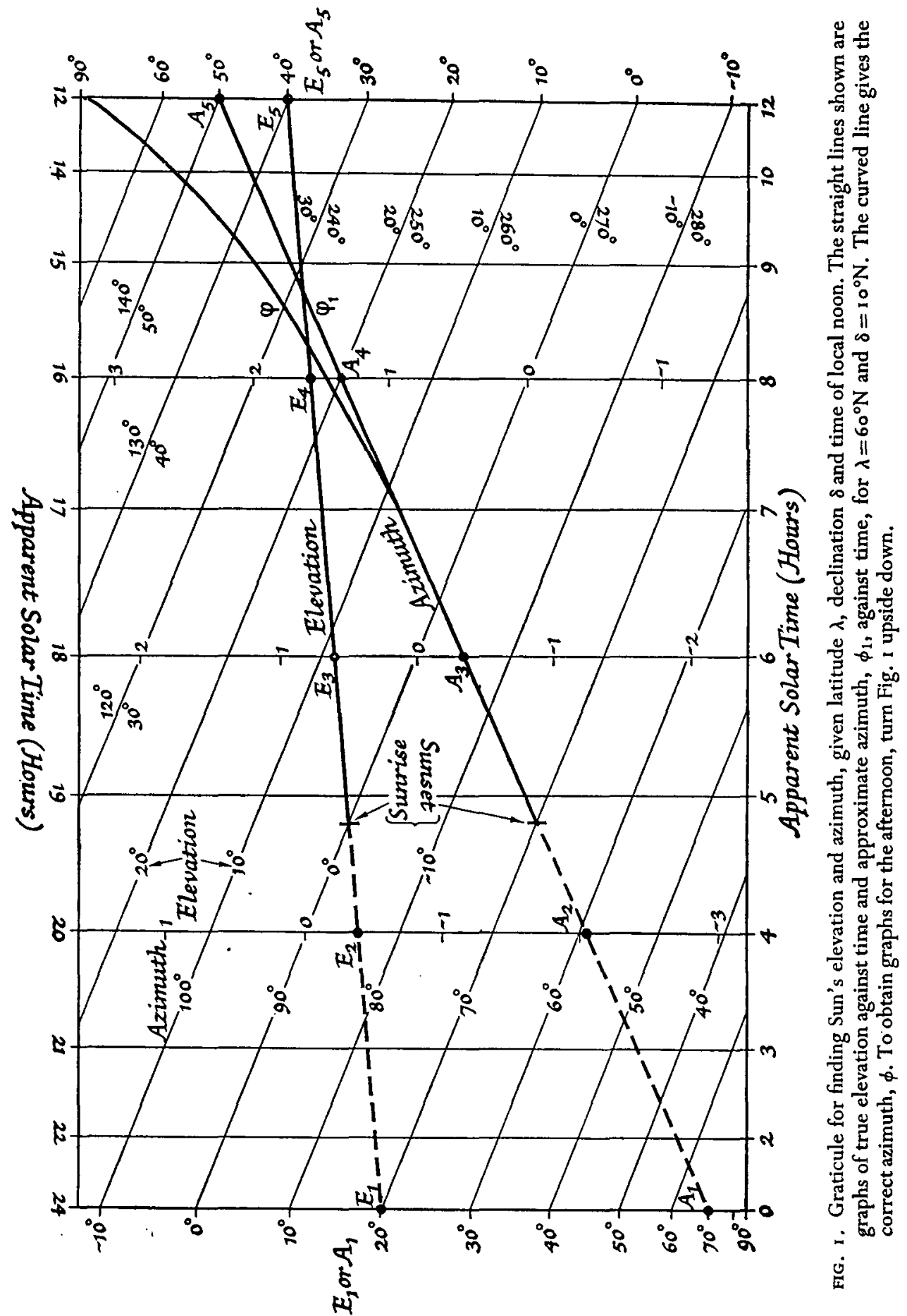


carry out the computation and construction as above, very simply on his own. The only apparatus required is the graticule, a table of sines and a long straight edge; the computation consists entirely of addition, subtraction and one simple multiplication.

The way in which the method. works can be explained by stating that $E_{1}$ gives the elevation of the Sun below the horizon at midnight, $\mathrm{E}_{5}$ gives the elevation above the horizon at midday, and the scales of the graticule have been adjusted so that the graph between $E_{1}$ and $E_{5}$ is a straight line; the other points, $E_{2}, E_{3}$ and $E_{4}$, are merely the points a quarter, a half and three-quarters of the way along the straight line; it is not strictly necessary to compute and enter these three points, but they do provide a graphical check, and some improvement in accuracy.

The construction is based on the equation

$$
\sin \theta_{t}=\sin \delta \sin \lambda+\cos \delta \cos \lambda \cos H
$$

where $\theta_{t}=$ true elevation

and $H=$ local hour angle $\left(=15^{\circ} \times\right.$ (apparent solar time -12 hours)).

It follows from this equation that the graph of $\sin \theta_{t}$ against $\cos H$ is a straight line. We therefore choose $x=1+\cos H$ and $y=\sin \theta_{t}$ as oblique Cartesian coordinates and construct the graticule of Fig. I accordingly.

At the point $\mathrm{E}_{1}, H=180^{\circ}, \cos H=-\mathrm{I}$, hence $\sin \theta_{t}=-\cos (\lambda+\delta)$ and $\theta_{t}=-\left(90^{\circ}-\lambda-\delta\right)$.

At the point $\mathrm{E}_{5}, H=0^{\circ}, \cos H=\mathrm{I}$, hence $\sin \theta_{t}=\cos (\lambda-\delta)$ and $\theta_{t}=90^{\circ}-\lambda+\delta$. Since the graph of $\sin \theta_{t}$ against $\cos H$ is a straight line, and these two points lie on it, this graph must be the line $E_{1} E_{5}$.

Generally, the radio engineer wishes to know the apparent elevation, $\theta_{a}$, which exceeds the true elevation, $\theta_{t}$, by the amount shown in Fig. 2. If desired,

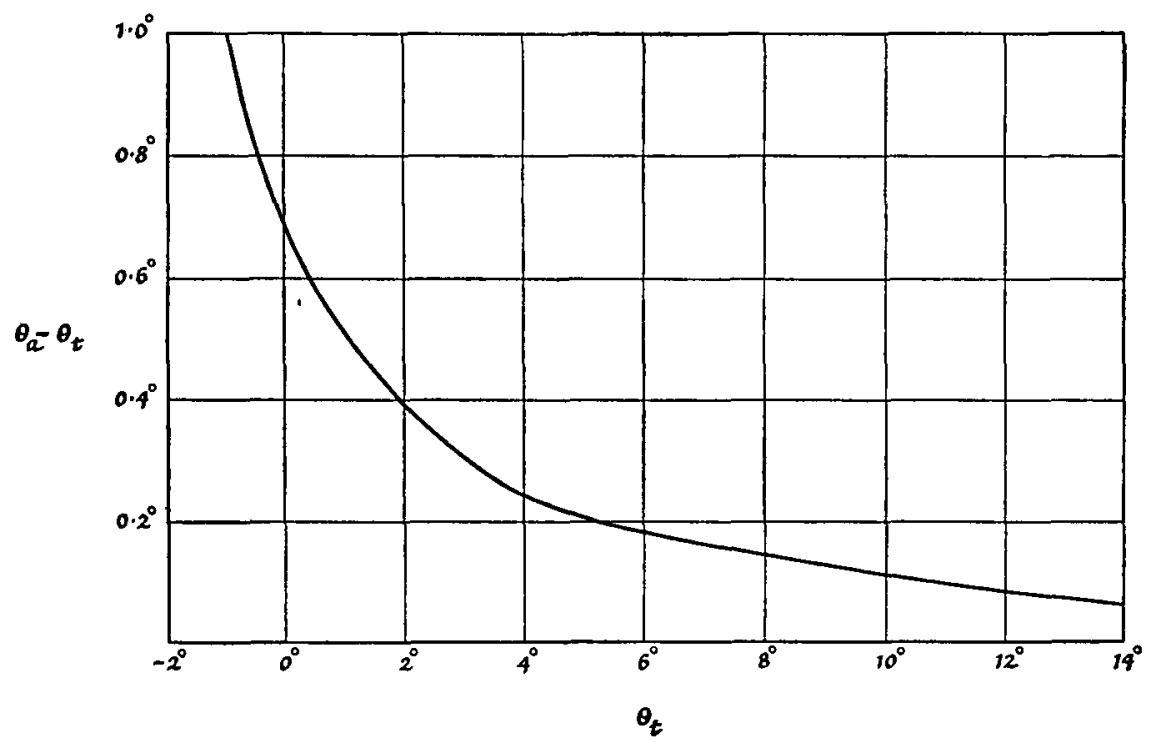

FIG. 2. Mean refraction of radio waves by the atmosphere, $\theta_{t}=$ true elevation, $\theta_{a}=$ apparent elevation. 


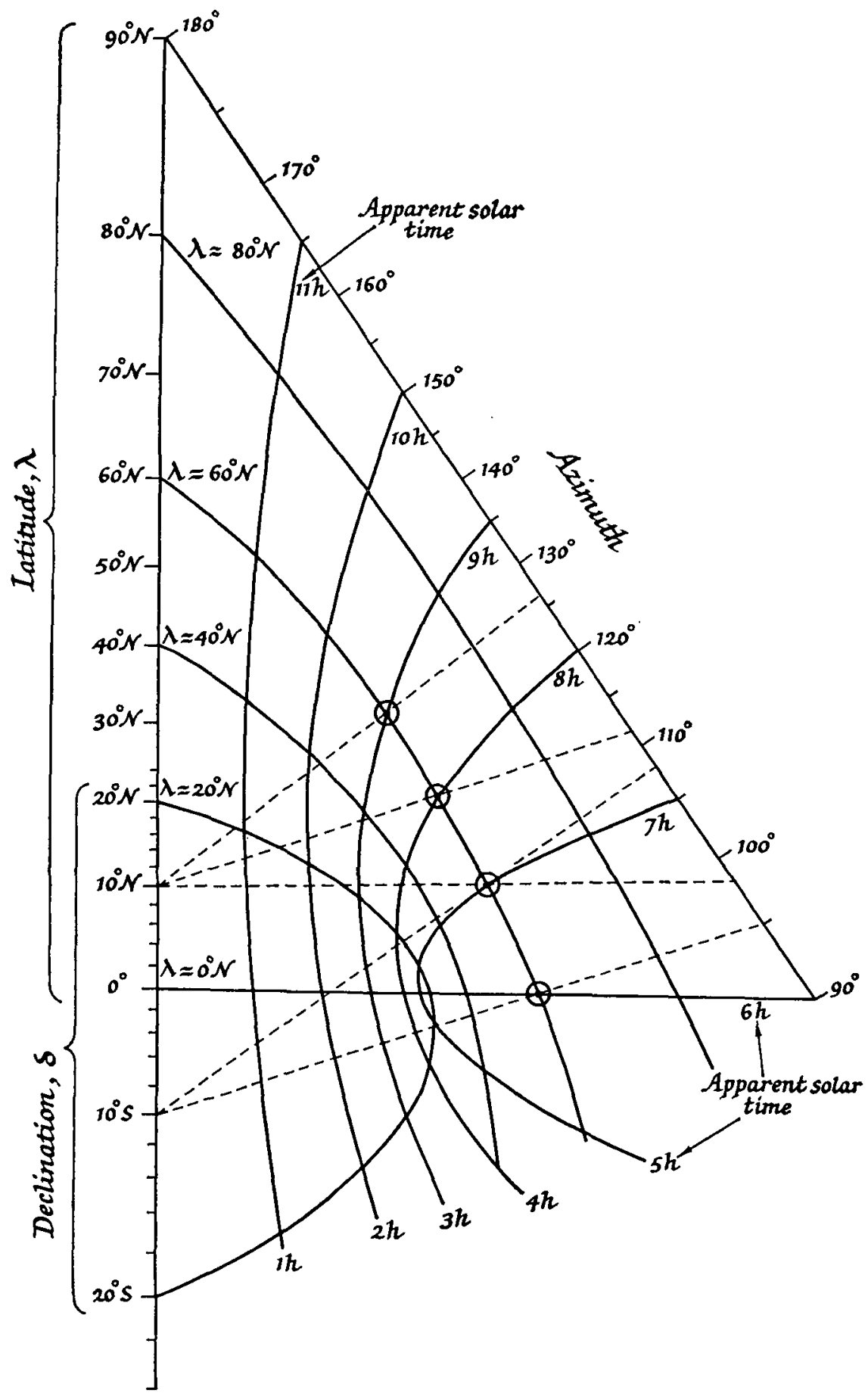

FIG. 3. Nomogram for finding the azimuth, $\phi$, in the morning in the northern hemisphere. When $\phi<90^{\circ}$, enter the contrary declination and 12 hours-apparent solar time; the intercept is then $180^{\circ}$-azimuth. The dashed lines indicate the following azimuths for $\lambda=60^{\circ} \mathrm{N}, \quad \delta=10^{\circ} \mathrm{N}$.

\begin{tabular}{l|c|c|c|c|c}
\hline Time & 05.00 & 06.00 & 07.00 & 08.00 & 09.00 \\
\hline Azimuth & $72^{\circ}$ & $85^{\circ}$ & $98^{\circ}$ & $111 \frac{1}{2}^{\circ}$ & $126 \frac{1}{2}^{\circ}$ \\
\hline
\end{tabular}


a graticule showing $\theta_{a}$ can be superimposed on Fig. I, and from this graticule the apparent elevation can be read directly.

There are two alternative graphical methods of finding azimuth. The nomogram shown in Fig. 3 is universally applicable, but its accuracy varies with latitude and declination and nowhere exceeds about $\pm 0:_{3}$ (for a nomogram 6 in. wide). It is based on the equation

$$
\cot \phi=\sin \lambda \cot H-\tan \delta \cos \lambda \operatorname{cosec} H
$$

where $\phi=$ azimuth.

It is possible to insert on Fig. I a graph giving azimuth. This method is based on the equations

and

$$
\cos \phi=\cos \phi_{1} \sec \theta_{t}
$$

$$
\cos \phi_{1}=\cos \lambda \sin \delta-\sin \lambda \cos \delta \cos H
$$

It follows from the first of these equations that when $\theta_{t}$ is small the value of $\phi$ is nearly the same as that of $\phi_{1}$. It also follows from the second equation that the graph of $\phi_{1}$ on the graticule of Fig. I is a straight line (by putting $y$ on the graticule equal to $-\cos \phi_{1}$ ). The points $A_{1}$ to $A_{5}$ on this straight line are computed as follows:

$$
\begin{aligned}
\mathrm{A}_{1} & =\lambda+\delta \\
\mathrm{A}_{5} & =\lambda-\delta \\
C & =\sin \mathrm{A}_{1} \\
D & =\sin \mathrm{A}_{5} \\
N & =C+D \\
\mathrm{~A}_{2} & =-4 C+N \\
\mathrm{~A}_{3} & =-4 C+2 N \text { or } 2(D-C) \\
\mathrm{A}_{4} & =-4 C+3 N
\end{aligned}
$$

The value of $\phi$ can be found from that of $\phi_{1}$ and $\theta_{t}$ either from equation (3) or by means of a diagram such as Fig. 4. The ringed point in this diagram indicates, for example, that when $\phi_{1}=60^{\circ}$ and $\theta_{t}=10^{\circ}$, then $\epsilon\left(=\left|\phi-\phi_{1}\right|\right)$ is $0^{\circ} 5$. It is clear from equation (3) that $\phi$ always gives a point nearer to north or south than $\phi_{1}$. Hence if $\phi_{1}=60^{\circ} \circ$, then $\phi=59^{\circ} 5$; if $\phi_{1}=1200^{\circ}$, then $\phi=120^{\circ} 5$, and so on.

It is noteworthy that the difference between $\phi$ and $\phi_{1}$ is small over a considerable range of values of $\phi_{1}$ and $\theta_{t}$. This can be seen clearly in Fig. I. For example, if an error in azimuth of $\pm 1^{\circ}$ is tolerable, then the graph of $\phi_{1}$ in Fig. 1 may be taken to give azimuth directly at all times between sunrise and $\circ 7 \mathrm{~h}$. $3 \circ \mathrm{m}$. (elevation $20^{\circ}$ ); this may be sufficient for all radio purposes.

The graphical methods described above are, like all other graphical methods, of limited accuracy, but their simplicity may well prove advantageous whenever the azimuth and/or elevation of an astronomical body is required to an accuracy no better than $\pm 0^{\circ} \mathrm{I}$. In fact, provided that sufficiently accurate graticules and nomograms like Figs. I, 3 and 4 are available, these graphical methods may well prove to be the most straightforward of all possible methods for engineers to use in the field. As an alternative method, a programme has now been written for an automatic computer, which finds azimuth and elevation at equal intervals of time (e.g. every 20 minutes) for a given location and declination.

In order to use this programme, it is necessary to punch input cards, to put these and the programme cards into the machine, to transfer the output cards to 


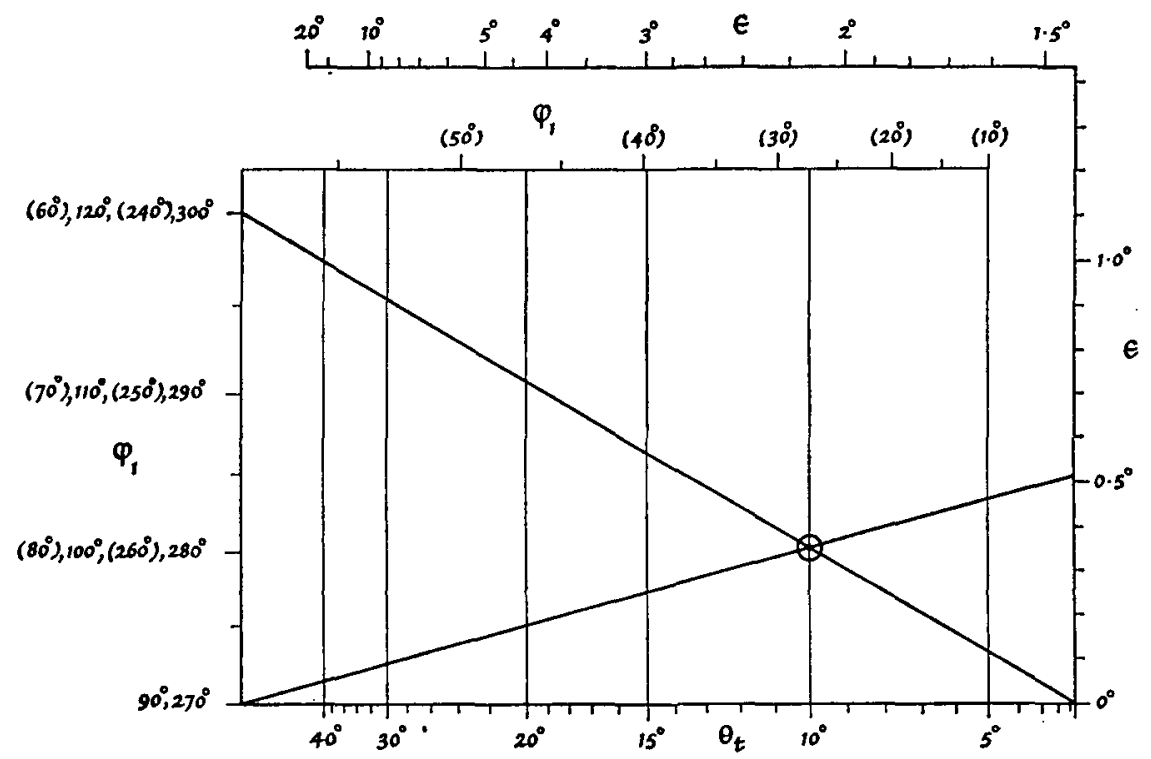

FIG. 4. Graticule for finding $\epsilon=\left|\phi-\phi_{1}\right|$

a tabulator, and to rewrite the tabulated results in a form suitable for the engineer. The total labour involved in this procedure is actually greater than the total labour required for a graphical computation. It appears therefore that the graphical methods will normally be used by engineers, and that machine or other methods will be used only for special purposes. For example, if many values are required to an accuracy better than $\pm 0^{\circ} 1$, it is probably best to use the machine; if only one or two accurate values are required, then it may be advisable to use ordinary hand computation, with or without the aid of tables of computed azimuth and altitude. The graphical methods will, however, always have the advantage of being simple to use and self-checking.

The construction can also be used for solving other problems in spherical trigonometry. For example, the elevation and azimuth graphs in Fig. I give, respectively, the latitude and longitude of all places at which the elevation of a certain star at declination $60^{\circ} \mathrm{N}$. is $10^{\circ}$ (e.g. the star's elevation is $10^{\circ}$ when observed from a point at latitude $20^{\circ} \mathrm{N}$., longitude $105^{\circ}$ different from that of the star). The same figures give the locus of all points on the Earth which are $80^{\circ}$ from a certain point at latitude $60^{\circ}$. This type of information can be used to construct sunset or twilight contours, or contours of constant elevation of an artificial satellite. The elevation and azimuth graphs in Fig. I can also be used to give the distance and bearing respectively of points at latitude $10^{\circ} \mathrm{N}$. from points at latitude $60^{\circ} \mathrm{N}$., e.g. since Trinidad is $72^{\circ}$ west of Oslo, its distance from Oslo is $72^{\circ}(=4300 \mathrm{n} . \mathrm{m}$. or $8000 \mathrm{~km}$. $)$ and its bearing is $259^{\circ}$.

I would like to thank the Chief of Research of Marconi's Wireless Telegraph Co. Ltd. for permission to publish this note. 